\title{
Monodic ASMs and Temporal Verification ${ }^{\star}$
}

\author{
Michael Fisher and Alexei Lisitsa \\ Department of Computer Science, University of Liverpool, Liverpool L69 7ZF, UK \\ \{M.Fisher,A.Lisitsa\}@Csc.Iiv.ac.uk
}

\begin{abstract}
In this paper, we pursue the goal of automatic deductive verification for certain classes of ASM. In particular, we base our work on a translation of general ASMs to full first-order temporal logic. While such a logic is, in general, not finitely axiomatisable, recent work has identified a fragment, termed the monodic fragment, that is finitely axiomatisable and many of its subfragments are decidable. Thus, in this paper, we define a class of monodic ASMs whose semantics in terms of temporal logic fits within the monodic fragment. This, together with recent work on clausal resolution methods for monodic fragments, allows us to carry out temporal verification of monodic ASMs. The approach is illustrated by the deductive verification of FloodSet algorithm for Consensus problem, and Synapse $\mathrm{N}+1$ cache coherence protocol; both are specified by monodic ASMs.
\end{abstract}

\section{Introduction}

The underlying idea which provides Abstract State Machines (ASMs) with much of their flexibility, is that states of an arbitrary computation are represented by general many-sorted first-order structures. The vocabularies of these structures may be freely chosen in order to model any particular algorithm at an appropriate level of abstraction [16]. (This flexibility contrasts with many standard models, such as Turing machines or finite state automata, where the alphabets used are fixed.) The process of computation is then modelled by the evolution of states according to given rules; on being 'fired', these rules change the structure produced by re-interpreting its basic functions.

Since its introduction, the ASM approach has proved to be very successful in specifying a variety of different types of software and hardware, including abstract algorithms, realistic programming languages, and distributed computations [1]. This success provides empirical evidence supporting the Abstract State Machine Thesis [16], which asserts that every algorithm is behaviourally equivalent to an ASM and so may be simulated, step-by-step, by that machine. For example, in [17] and [2], this thesis was established for very general formalisations of the notions of sequential and parallel algorithms, respectively. The combination of generality, varying levels of abstractness and strong formal foundations has led to the ASM approach beginning to be applied in significant industrial applications [18-20].

However, while specification and implementation techniques using ASMs have been studied in depth, there has been relatively little work on the verification of ASMs.

\footnotetext{
* The authors acknowledge partial support from EPSRC (through grants GR/M46631 and GR/R45376) for the work reported in this paper.
} 
Research in this area has, so far, mainly concerned either human-assisted proofs of correctness $[36,15]$, dealing with ASM representations in a relatively manual way, or algorithmic verification of ASMs [3, 9, 38, 39], based on model checking techniques [5].

In this paper, we pursue the goal of automatic deductive verification for certain classes of ASM. In particular, we base our work on a translation of general ASMs to full first-order temporal logic with natural numbers as a time flow [11]. While such a logic is, in general, not finitely axiomatisable, recent work has identified a fragment, termed the monodic fragment, that is finitely axiomatisable [23], thus making a semi-decision procedure possible. Furthermore, some of its natural (e.g two variables or monadic) subfragments, are decidable. Thus, in this paper, we define a class of monodic ASMs whose semantics in terms of temporal logic fits within the monodic fragment. This, together with recent work on clausal resolution methods [12] for monodic fragments [6, $28,7]$, allows us to carry out temporal verification of properties of monodic ASMs. The approach is illustrated by the deductive verification of the correctness of both a FloodSet algorithm for the Consensus problem (in the presence of crash failures) and a cache coherence protocol, both specified using monodic ASMs.

We begin, in Sections 2 and 3 respectively, with descriptions of the ASM approach and the variety of temporal logic we use. In Section 4, we define how general ASMs can be translated into formulae in first-order temporal logic, and establish formal properties of such a translation. Given that a translation of general ASMs to temporal logic is possible, we next consider, in Section 5, what class of ASMs translate to monodic first-order temporal logic. We define the class of monodic ASMs that capture this property, thus allowing systems specified in this class to be translated into a temporal fragment amenable to automatic (or at least semi-automatic) verification. In Section 6, we consider two applications: one concerning a distributed consensus problem; the other concerning cach coherence protocols. In Section 7 we compare our work with related work of A.Nowack. Finally, in Section 8, we provide concluding remarks and discuss future work.

\section{Abstract State Machines}

\section{Definition 1}

An Abstract State Machine, $\mathcal{M}$, can be defined as a tuple $\mathcal{M}=\left\langle\sigma, T R, I C, \Psi_{0}\right\rangle$ where:

- $\sigma$ is a many-sorted signature, i.e. a finite collection of function names, together with their sorts; $\sigma$ is a disjoint union of three (possibly empty) parts: $\sigma=\sigma_{s} \cup \sigma_{d} \cup \sigma_{e}$, which consist of static, dynamic and external names, respectively. We assume the static part, $\sigma_{s}$ includes constants for all elements of any finite sort. We will identify boolean-valued terms with predicates whenever convenient.

- TR is a finite set of transition rules, i.e. the program of $\mathcal{M}$, where a transition rule is an expression of the form

$$
\text { if } g(\bar{x}) \text { then } U_{1}(\bar{x}), \ldots U_{m}(\bar{x})
$$

where $g(\bar{x})$ is a first-order formula (a guard) of the given signature over variables $\bar{x}$ and $U_{i}(\bar{x})$ is an expression of the form $f\left(t_{1}(\bar{x}), \ldots, t_{k}(\bar{x})\right)=t(\bar{x})$ where $f \in \sigma_{d}$ and $\bar{x}$ are all free variables of the update, although any term $t_{1}, \ldots t_{k}, t$, or guard $g(\bar{x})$ is not required to contain all of $\bar{x}$; 
- IC is a set of integrity constraints, with each being a first-order sentence within $\sigma_{s} \cup \sigma_{e}$;

- $\Psi_{0}$ is a first-order sentence in the vocabulary $\sigma$ describing initial condition.

The idea underlying the separation of $\sigma$ into three parts is as follows. The static names, i.e. those from $\sigma_{s}$ are assumed to not change their interpretations during the computation. The interpretations of dynamic names $\left(\sigma_{d}\right)$ may change during the computation by the application of transition rules from $T R$. External names $\left(\sigma_{e}\right)$ serve to model the interaction with the environment and their interpretations are not subject to change by transition rule applications, but nevertheless their interpretations may be different in different states. It is natural to consider the evaluation of an external function as being carried out by an oracle (environment).

Note 1. Our definition is somewhat more general than the common one, where instead of the sentence $\Psi_{0}$ an initial first-order structure $S_{0}$ is assumed.

Note 2. We do not allow dynamic functions whithin integrity constraints. There are two reasons for this. First, this would make the definition of the operational semantics more complicated. Second, one can model any possible effects of such constraints by appropriate extension of the transition rules. We feel, however, that in some cases constraints including dynamic functions are more natural and we are plan to consider these elsewhere.

Definition 2 A state of an ASM $\mathcal{M}=\left\langle\sigma, T R, I C, \Psi_{0}\right\rangle$ is a (many-sorted) first-order structure in a signature $\sigma$

Definition 3 A computation (run) of an ASM $\mathcal{M}$ is a sequence of its states $S_{0}, \ldots S_{k}, \ldots$ such that

- $S_{0} \models \Psi_{0}$, i.e. the initial state satifies the initial condition.

- the domain of each $S_{i}$ coincides with that of $S_{0}$;

- the interpretations of all functional symbols from $\sigma_{s}$ in each $S_{i}$ coincide with those in $S_{0}$;

- the interpretation of all functional symbols from $\sigma_{d}$ in $S_{i+1}$ is obtained from the state $S_{i}$ by applying of all transition rules, subject to the restrictions that every $S_{i}$ satisfies all the integrity constraints from $I C$ and the updates are consistent in $S_{i}$. The application of a transition rule within a given state $S_{i}$ of $\mathcal{M}$ means simultaneously executing the updates $U_{1}(\bar{x}), \ldots, U_{m}(\bar{x})$ over the state $S_{i+1}$ whenever the guard, $g(\bar{x})$ is satisfied. Thus, if an update $f\left(t_{1}(\bar{x}), \ldots, t_{k}(\bar{x})\right)=t(\bar{x})$ is executed in $S_{i}$ then $f^{S_{i+1}}\left(t_{1}^{S_{i}}(\bar{x}), \ldots, t_{k}^{S_{i}}(\bar{x})\right)=t^{S_{i}}(\bar{x})$ holds for all $\bar{x}$ such that $S_{i}=g(\bar{x})$. The consistency of updates in a state $S$ means that no two different updates are to update differently the interpretation of same function on the same argument.

- the interpretation of all functional symbols from $\sigma_{e}$ may be arbitrary, provided every $S_{i}$ satisfies all the integrity constraints from $I C$.

Definition 4 If, at some state $S$, the updates are inconsistent, or execution of updates leads to the state violating some integrity constraint, it is said that $\mathrm{M}$ crashes in $S$. For example, the rules if $b$ then $f(3)=5$ and if $b$ then $f(3)=7$ would lead to the crash of the machine in a state $S$ such that $S \models b$. 
Note 3. According to the Definition 3, any run is either infinite or a finite crashing one. This is essential in our approach since we will be using first-order temporal logic over an infinite flow of time isomorphic to $\omega$ to model an execution of ASMs. However, it does not prevent one dealing with finite runs of ASMs, ending by reaching some end condition (not by crashing) within this approach: one may formally extend the finite runs into infinite ones by adding obvious rules to ASM forcing the machine to loop over last state forever.

Note 4. We consider basic and restricted forms of ASM. The most notable omission is import instructions, allowing new elements to be imported to the domain during of computation [16]. In addition, we do not consider other extensions of ASM such as non-deterministic choice, object-orientation, or distribution mechanisms [1].

Definition 5 An ASM rule is said to be normal if, and only if, it consists of at most one update in its right-hand side. An ASM program is normal if, and only if, it consists of only normal rules.

Proposition 1. Any ASM program can be transformed into an equivalent normal ASM program.

Proof. Simply replace any rule if $g(\bar{x})$ then $U_{1}(\bar{x}), \ldots, U_{m}(\bar{x})$ with the set of rules $\left\{\right.$ if $g(\bar{x})$ then $\left.U_{i}(\bar{x}) \mid i=1 \ldots m\right\}$.

\section{Temporal Logic}

The representation of dynamic activity via temporal formalisms is used in a wide variety of areas within Computer Science and Artificial Intelligence, for example temporal databases, program specification, system verification, agent-based systems, robotics, simulation, planning, knowledge representation, and many more [31, 32, 5, 40, 27].

\subsection{First-Order Temporal Logic (FOTL)}

Here, we concentrate on one very popular variety, namely discrete linear temporal logic, which has an underlying model of time isomorphic to the Natural Numbers (i.e. an infinite sequence with distinguished initial point) and is also linear, with each moment in time having at most one successor. The infinite and linear constraints ensure that each moment in time has exactly one successor.

The language $\mathcal{T L}$ of the first order temporal logic over the Natural Numbers is constructed in the standard way from a classical (non-temporal) first order language $\mathcal{L}$ and a set of future-time temporal operators ' $\nabla$ ' (sometime), ' $\square$ ' (always), ' $\bigcirc$ ' (in the next moment), ' $U$ '(until).

Formulae in $\mathcal{T} \mathcal{L}$ are interpreted in first-order temporal structures of the form $\mathfrak{M}=$ $\langle D, I\rangle$, where $D$ is a non-empty set, the domain of $\mathfrak{M}$, and $I$ is a function associating with every moment of time $n \in \mathbb{N}$ an interpretation of predicate, function and constant symbols of $\mathcal{L}$ over $D$. First-order (nontemporal) structures corresponding to each point of time will be denoted $\mathfrak{M}_{n}=\langle D, I(n)\rangle$. Intuitively, the interpretations of $\mathcal{T} \mathcal{L}$-formulae 
are sequences of first-order structures, or states of $\mathfrak{M}$, such as $\mathfrak{M}_{0}, \mathfrak{M}_{1}, \ldots, \mathfrak{M}_{n} \ldots$ An assignment in $D$ is a function $\mathfrak{a}$ from the set $\mathcal{L}_{\mathbf{v}}$ of individual variables of $\mathcal{L}$ to $D$. If $P$ is a predicate symbol then $P^{I(n)}$ (or simply $P^{n}$ if $I$ is undersood) is the interpretation of $P$ in the state $\mathfrak{M}_{n}$. We require that (individual) variables and constants of $\mathcal{T} \mathcal{L}$ are rigid, that is neither assignments nor interpretations of constants depend on the state in which they are evaluated. On the other hand we allow both predicates and functional symbols to be flexible. Flexibility of functions, which is not a very common assumption in the semantics of FOTL, is very convenient for direct modelling of ASM rules.

The truth-relation $\mathfrak{M}_{n} \models^{\mathfrak{a}} \varphi$ (or simply $n \models^{\mathfrak{a}} \varphi$, if $\mathfrak{M}$ is understood ) in the structure $\mathfrak{M}$ for the assignment $\mathfrak{a}$ is defined inductively in the usual way under the following semantics of temporal operators:

$$
\begin{aligned}
& n \models^{\mathfrak{a}} \bigcirc \varphi \text { iff } n+1 \models^{\mathfrak{a}} \varphi ; \\
& n \models^{\mathfrak{a}} \nabla \varphi \text { iff there is } m \geq n \text { such that }\left.m\right|^{\mathfrak{a}} \varphi ; \\
& n \models^{\mathfrak{a}} \square \varphi \text { iff } m \models^{\mathfrak{a}} \varphi \text { for all } m \geq n ; \\
& n \models^{\mathfrak{a}} \varphi \mathcal{U} \psi \text { iff there is } m \geq n \text { such that }\left.m\right|^{\mathfrak{a}} \psi \text { and } \\
& \quad k \models^{\mathfrak{a}} \varphi \text { for every } n \leq k<m ;
\end{aligned}
$$

A formula $\varphi$ is said to be satisfiable if there is a first-order structure $\mathfrak{M}$ and an assignment $\mathfrak{a}$ such that $\mathfrak{M}_{0} \models{ }^{\mathfrak{a}} \varphi$. If $\mathfrak{M}_{0} \models{ }^{\mathfrak{a}} \varphi$ for every structure $\mathfrak{M}$ and for all assignments then $\varphi$ is said to be valid. For a closed formula $\varphi$ denote by $\operatorname{Mod}(\varphi)$ the set of all its models, i.e. first-order temporal structures $\mathfrak{M}$ such that $\mathfrak{M}_{0} \models \varphi$.

\subsection{Monodic FOTL}

While first-order temporal logic (FOTL) is very expressive and, in general, highly undecidable, recent results have identified recursively enumerable fragments of FOTLs [23].

Definition 6 A first-order temporal formula is said to be monodic if, and only if, any subformula with its main connective being a temporal operator has at most one free variable.

The monodic fragment of FOTL has appealing properties. It is axiomatizable [41] and many of its subfragments, such as the two-variable or monadic cases, are decidable. Decidability of these fragments holds also for the case of semantics where only temporal structures over finite domains are allowed. However, these good properties hold mainly for the case when equality is not allowed. Adding equality makes even very restricted fragments non-axiomatizable [8]. A notable exception is the packed monodic fragment with equality, which is decidable [22].

\subsection{Temporal Tools}

The widespread use of temporal logics has led to the development of key techniques for utilising temporal specifications, such as model checking and deductive verification [30, 12]. While the former has restrictions in terms of the size of models considered, the latter has problems in terms of worst-case complexity. For propositional (discrete, linear) temporal logics, the decision procedure is already PSPACE [37]. In spite of this, a range 
of useful tools have been developed, with our clausal resolution approach [12] having been shown to be particularly effective [26].

For general (again, discrete and linear) FOTL, incompleteness is a problem. While there have been formalisations of proof mechanisms, for example infinitary sequent systems, proof in such complex logics will require significant user interaction. We have developed a prover, based on the $\lambda$ Clam proof planning system [35] which incorporates techniques from the clausal resolution method in order to aid proof search [4].

The breakthrough concerning monodic FOTL is now leading to the development of improved proof methods. We have extended our clausal resolution approach to this fragment [6, 28,7], and have also provided usable implementations [24]. We believe this to be a most productive route for temporal verification in the future.

\section{From ASM to FOTL}

In this paper, FOTL provides the key tool for formalising the execution of ASMs. In particular, as the operational semantics of an ASM is defined in terms of runs (sequences, or partially-ordered sets, of states), these can naturally be seen as the models of FOTL.

\begin{tabular}{|c|c|}
\hline$[[\mathcal{M}]]$ & $\begin{aligned}= & {\left[\left[\Psi_{0}\right]\right]_{\text {initial }}^{\sigma} \wedge[[\sigma]]_{\text {sorts }} \wedge[[\sigma]]_{\text {static }} \wedge[[T R]]_{\text {rules }}^{\sigma} \wedge } \\
& \wedge\left[[I C]_{\text {integrity }}^{\sigma} \wedge[[T R]]_{\text {frame }}^{\sigma}\right.\end{aligned}$ \\
\hline$\left[\left[\Psi_{0}\right]\right]_{\text {initial }}^{\sigma}$ & $=\left[\left[\Psi_{0}\right]\right]_{f o}^{\sigma}$ \\
\hline$[[\sigma]]_{\text {static }}$ & $=\square \bigwedge_{f \in \sigma_{s}} \forall \bar{x}, y$ Persists $(f(\bar{x})=y)$ \\
\hline$\left[\left[\left\{r_{1}, r_{2}, \ldots, r_{n}\right\}\right]\right]_{\text {rules }}^{\sigma}$ & $=\bigwedge_{i=1}^{n}\left[\left[r_{i}\right]_{\text {rule }}^{\sigma}\right.$ \\
\hline$\left[\left[\left\{c_{1}, c_{2}, \ldots, c_{n}\right\}\right]\right]_{\text {integ } r}^{\sigma}$ & $=\square \bigwedge_{i=1}^{n}\left[\left[c_{i}\right]_{f o}^{\sigma}\right.$ \\
\hline
\end{tabular}

Fig. 1. ASM $\rightarrow$ FOTL Translation Functions

\subsection{Translation}

We assume in this section that an ASM program is given in a normal form. In Fig. 1, we present translation functions which effectively provide a semantics in terms of firstorder temporal logic for an ASM, ' $\mathcal{M}$ '. In the Figure and below we use the notation Persists $(\varphi(\bar{x}))$ to denote $\varphi(\bar{x}) \rightarrow \bigcirc \varphi(\bar{x})$. Four semantic functions remain undefined and require further explanation.

First-Order Translation. The $[[]]_{f o}^{\sigma}$ function simply takes the many-sorted first order description given as an argument and produces a first-order description to be used in the temporal semantics. In the case of general ASMs considered here, this is simply the identity function. Later, however, we will see that some modification of the form of 
the first-order sentences can be useful when translating from ASM notation to FOTL formulae.

Sort Constraints. The $[[\sigma]]_{\text {sorts }}$ function takes the vocabulary $\sigma$ and produces $(\square$ closed) one-sorted first-order description of all sort constraints assumed for many-sorted functional symbols. For finite sorts, it also contains sentences enumerating explicitely the elements of these sorts.

Translation of Rules. Before we present the general translation of rules, we will consider an example. Translating the ASM transition rule, if $b(x)$ then $f(3, k(x))=2$, we have to take into account that the term $k(x)$ and the update $f(3, k(x))=2$ according to the semantics of ASM rules, should be evaluated in different time slices. So, a straighforward translation $\square \forall x(b(x) \rightarrow \bigcirc f(3, k(x))=2)$ would be incorrect. The correct translation is

$$
\square \forall x \forall v[(b(x) \wedge(v=k(x)) \rightarrow \bigcirc f(3, v)=2]
$$

In general, given any rule $r$ : if $g(\bar{x})$ then $f\left(t_{1}(\bar{x}), \ldots, t_{k}(\bar{x})\right)=t(\bar{x})$, we define the formula

$$
\Phi_{r}\left(\bar{x}, v_{1}, \ldots, v_{k}\right) \leftrightarrow g(\bar{x}) \wedge \bigwedge_{i=1 \ldots k}\left(v_{i}=t_{i}(\bar{x})\right)
$$

where all $v_{i}$ are fresh variables. Then we define the translation $\left[[r]_{\text {rule }}^{\sigma}\right.$ of $r$ as

$$
\square\left[\forall \bar{x}, \bar{v}, z\left(\Phi_{r}(\bar{x}, \bar{v}) \wedge z=t(\bar{x}) \rightarrow \bigcirc f(\bar{v})=z\right)\right]
$$

where $z$ is a fresh variable.

Frame Conditions. In addition to the transition rules and integrity constraints that are explicitly part of the ASM notation, there are implicit frame conditions. In essence, these conditions aim to ensure that any function retains its previous value on any argument if not affected by an update (or integrity constraint). Thus, the formula produced by $\left[[T R]_{\text {frame }}^{\sigma}\right.$ attempts to capture this persistence. The approach we follow in generating this formula is based on the simple idea provided by Reiter [34], namely that all the potential updates and guards within transition rules are examined and new formulae are added to describe the behaviour if one or more of the guards are not satisfied.

Consider as an example the ASM program with the following transition rules:

$$
\begin{aligned}
& \text { if } b(x) \text { then } f(3, k(x))=2 \\
& \text { if } c(x) \text { then } f(x, 5)=7 \\
& \text { if } d \text { then } f(4, m(x))=3
\end{aligned}
$$

Given some state $s$ we are interested in identifying those elements $v$ and $w$ of the domain of $s$, for which $f(v, w)$ will not change its value after executing the above rules in $s$. It is clear that this is the case precisely when $\langle v, w\rangle$ is not a pair of values of arguments of $f$ in the left-hand side of any of the updates under substitution which makes the corresponding guard true. Now, for the above transition rules, we have the following frame condition (where variables $v, w$, and $z$ are universally quantified and the whole formula is assumed to be under "always" operator): 


$$
\left(\begin{array}{c}
\neg \exists x(v=3 \wedge w=k(x) \wedge b(x)) \\
\wedge \\
\neg \exists x(x=v \wedge w=5 \wedge c(x)) \\
\wedge \\
\neg \exists x(v=4 \wedge w=m(x) \wedge d)
\end{array}\right) \Rightarrow \operatorname{Persists}(f(v, w)=z)
$$

So, more generally, we define $\left[[T R]_{\text {frame }}^{\sigma}\right.$ as follows. For a function symbol $f$ define $I_{f}=\left\{i \mid r_{i} \in T R\right.$ and $r_{i}$ updates $\left.f\right\}$. Then, let $\left[[T R]_{\text {frame }}^{\sigma, f}\right.$ be

$$
\square \forall \bar{v}, z\left[\left(\bigwedge_{i \in I_{f}} \neg \exists \bar{x} \Phi_{r_{i}}(\bar{x}, \bar{v})\right) \rightarrow \operatorname{Persists}(f(\bar{v})=z)\right]
$$

where $\Phi_{r}$ is defined above (1). Finally, we define $\left[[T R]_{\text {frame }}^{\sigma}\right.$ as $\bigwedge_{f \in \sigma_{d}}\left[[T R]_{\text {frame }}^{\sigma, f}\right.$

\subsection{Correctness of the Translation}

Here, we give the general statement of correctness of the translation of ASMs into full (i.e. including equality) FOTL. This is based on the strong correspondence between the execution sequences of ASMs, and the models of FOTL. The operational semantics of sequential ASMs is defined in terms of runs i.e. sequences of first-order structures, which allows to consider the infinite runs as the models for discrete, linear first-order logic. The restriction to infinite runs is essential since we consider FOTL over an infinite flow of time isomorphic to $\omega$. (See also Note 3.)

Theorem 1 (Correctness of Translation) The set $\operatorname{Runs}(\mathcal{M})$ of infinite runs of an ASM, $\mathcal{M}$, is exactly the set of models $\operatorname{Mod}([[\mathcal{M}]])$ of the formula $[[\mathcal{M}]]$, as defined in Fig. 1 and Section 4.1

Proof [Outline]. Given $\mathcal{M}=\left\langle\sigma, T R, I C, \Psi_{0}\right\rangle$, then Fig. 1 defines

$$
[[\mathcal{M}]]=\left[\left[\Psi_{0}\right]\right]_{\text {initial }}^{\sigma} \wedge[[\sigma]]_{\text {static }} \wedge\left[[ T R ] _ { \text { rules } } ^ { \sigma } \wedge \left[[I C]_{\text {integrity }}^{\sigma} \wedge[[T R]]_{\text {frame }}^{\sigma}\right.\right.
$$

Consider an arbitrary infinite sequence $\gamma=S_{0}, S_{0}, \ldots S_{k}, \ldots$ of first-order structures in the vocabulary $\sigma$. Then straightforward check of the definitions of run and translation shows that $\gamma \in \operatorname{Runs}(\mathcal{M})$ if, and only if, $\gamma \in \operatorname{Mod}([[\mathcal{M}]])$. See more details in [14].

\subsection{Verification of ASM Translations}

Given an ASM program $\mathcal{M}$ one may use the above translation for verification of this program as follows: express correctness conditions by a first-order temporal formula $\psi$ and then check whether the implication $[[\mathcal{M}]] \rightarrow \psi$ is a valid FOTL formula. In general, of course we have no decision, or even semi-decision procedure to check validity (or satisfiability) of such formulae. But, if the resulting formula turns out to be in a 'good' (e.g. monodic) fragment one can apply decision procedures, or theorem proving, for verification. Otherwise, one can apply heuristic methods to show its validity. Some such methods, derived from the resolution procedures for monodic fragments, and incomplete in general are presented in [4]. In what follows we describe restrictions on ASM programs that ensure their temporal translations are in monodic fragments. 


\section{From ASM to Monodic FOTL: Monodic ASMs}

In this section we are looking for restrictions of ASM programs which ensure that their temporal translations fall within "good" monodic fragments. A quick glance at the defined translations reveals that non-monodic formulae may appear both in the translations of transition rules and in frame conditions.

Definition 7 Any term which is not a constant or variable is said to be complex, otherwise it is simple.

Definition 8 A functional symbol $f$ of a sort $S_{1} \times \ldots \times S_{k} \rightarrow S$ within the ASM vocabulary is said to be finitely valued if, and only if, the sort $S$ is finite.

Definition 9 A normal transition rule, if $g(\bar{x})$ then $f\left(t_{1}(\bar{x}), \ldots, t_{k}(\bar{x})\right)=t(\bar{x})$ is called monodic if, and only if,

1. At most one free variable ranging over an infinite sort occurs both in the guard and in the update (i.e. the free variable is shared);

2. The main functional symbol $f$ of an update has at most one infinite input sort;

3. An immediate subterm $t_{i}(\bar{x})$ of $f\left(t_{1}(\bar{x}), \ldots, t_{k}(\bar{x})\right)$ of an infinite sort, is a variable (not a constant or complex term);

4. All functional symbols in the rule are finitely valued;

Definition 10 An ASM program is called monodic if, and only if,

- it consists of only normal monodic rules, and

- static part $\sigma_{s}$ of the vocabulary consists of only finitely valued functional symbols of arities at most 1 , and

- neither a guard of any rule, nor the initial condition of ASM nor integrity constraints contain equality.

Examples. Let $S_{i}$ be an infinite sort, $S_{f}$ be a finite sort and $a$ be a constant of sort $S_{f}$.

1. Assuming $g: S_{i} \times S_{i} \rightarrow S_{f}$, then the rule if true then $f(x, x)=a$ is not monodic (condition 2 does not hold)

2. Assume now that $g: S_{i} \times S_{f} \rightarrow S_{f}, k: S_{i} \rightarrow S_{f}$.

Then the rule if true then $g(x, k(x))=a$ is monodic.

\subsection{Translation of a Monodic ASM Program}

Before we formulate a general statement on the translation of monodic ASM programs into temporal logic, it is instructive to consider an example of such a translation. Take the transition rule if true then $f(x, k(x))=a$ of the previous example and consider an ASM program $\mathcal{M}$ which contains this as its only rule. Assume also $S_{f}=\{a, b\}$. Then the translation $\left[[T R]_{\text {rules }}^{\sigma}\right.$ of the rule is $\square \forall x, v(x=v \rightarrow \bigcirc(f(v, k(v))=a)$ and frame condition $[[T R]]_{\text {frame }}$ is $\forall v, w, z(\neg \exists x(v=x \wedge w=k(x)) \rightarrow \operatorname{Persist}(f(v, w)=z))$ which, after obvious simplification, become $\square \forall x \bigcirc(f(x, k(x))=a)$ and 


$$
\forall v, w, z(w \neq k(v) \rightarrow \operatorname{Persist} s(f(v, w)=z))
$$

Notice that the formula expressing the frame condition is not monodic: Persists is applied to the subformula with three free variables, which destroys monodicity. Further, both above translations contain functional symbols and equality, which also makes it problematic to be in any decidable, or axiomatizable, fragment of FOTL. However, replacing functional symbols with corresponding predicates and, further, using the finiteness conditions imposed by definition of a monodic ASM program, makes it possible to translate these formulae to the good monodic fragment, preserving the "meaning" of the original ASM program.

To replace functional symbols, we introduce new relational symbols (i.e. boolean valued functional symbols)

$$
\begin{aligned}
& \text { val }_{f}: S_{i} \times S_{f} \times S_{f} \rightarrow \text { Bool } ; \\
& \text { val }_{k}: S_{i} \times S_{f} \rightarrow \text { Bool }
\end{aligned}
$$

Then we can rewrite $\left[[T R]_{\text {rules }}^{\sigma}\right.$ into $\forall x \square \bigcirc\left(\exists u\left(\operatorname{val}_{k}(x, u) \wedge \operatorname{val}_{f}(x, u, a)\right)\right)$ which is now monodic and without functional symbols. Further, using the finiteness of the sort $S_{f}$ we can remove the existensial quantifier:

$$
\left[[T R]_{\text {rules }}^{*}=\forall x \square \bigcirc\left[\begin{array}{l}
\left(\left(\operatorname{val}_{k}(x, a) \wedge \operatorname{val}_{f}(x, a, a)\right) \vee\right. \\
\left(\operatorname{val}_{k}(x, b) \wedge \operatorname{val}_{f}(x, b, a)\right)
\end{array}\right]\right.
$$

Now, rewrite the frame condition into

$$
\forall v, w, z\left(\neg \operatorname{val}_{k}(v, w) \rightarrow \operatorname{Persist}\left(\operatorname{val}_{f}(v, w, z)\right)\right)
$$

Notice, that this is still a non-monodic formula. Denote it by $\forall v, w \Psi(v, w)$. Further, using finiteness of $S_{f}$ we can remove variables $v$ and $w$ and quantifiers upon them, making the resulting formula monodic:

$$
\left[[T R]_{\text {frame }}^{*}=\Psi(a, a) \wedge \Psi(a, b) \wedge \Psi(b, a) \wedge \Psi(b, b)\right.
$$

To finish this translation we have to specify the functionality property of newly introduced relational symbols. The corresponding formula $F u n_{k, f}$ :

$$
\forall x, y\left(\left(\operatorname{val}_{k}(x, a) \mid \operatorname{val}_{k}(x, b)\right) \wedge\left(\operatorname{val}_{f}(x, y, a) \mid \operatorname{val}_{f}(x, y, b)\right)\right)
$$

has to be added to the integrity constrains $[[I C]]_{\text {integrity }}$. Here $\mid$ stands for "exclusive OR".

Notice that $[[T R]]_{\text {rules }}^{*} \wedge\left[[T R]_{\text {frame }}^{*} \wedge F u n_{k, f}\right.$ is a monodic formula without functional symbols and equality. Notice also that it models closely the behaviour of the initial translation $\left[[T R]_{\text {rules }} \wedge\left[[T R]_{\text {frame }}\right.\right.$ : the sets of their temporal models are the same modulo renaming $f \mapsto v a l_{f}$ and $k \mapsto v a l_{k}$. Now we can formulate a general statement

Theorem 2 Let $\mathcal{M}$ be monodic ASM program. Then its temporal translation $[[\mathcal{M}]]$ can be further translated into monodic temporal formula $[[\mathcal{M}]]^{*}$ which:

- does not contain functional symbols;

- does not contain equality;

- has the same set of temporal models as $[[\mathcal{M}]]$ modulo some renaming of functional symbols into relational ones. 
Proof [OutLine]. Given a translation $\llbracket \mathcal{M} \rrbracket]$ of an ASM program $\mathcal{M}=\left\langle\sigma, T R, I C, S_{0}\right\rangle$ we perform further translation as follows. For every functional symbol $f$ of the sort $S_{1} \times \ldots \times S_{k} \rightarrow S$ introduce a corresponding predicate symbol val va $_{f}$ of the sort $S_{1} \times$ $\ldots \times S_{k} \times S \rightarrow$ Bool. For all functional symbols $f_{1}, \ldots, f_{s}$ add $F u n_{f_{1}, \ldots, f_{s}}$, the formulae expressing functionality of all $v_{a l} l_{f_{1}}, \ldots v a l_{f_{s}}$, to $\left[[I C]_{\text {integrity }}^{\sigma}\right.$. Because all of $f_{1}, \ldots f_{k}$ are finitely valued and there are constants for all elements of finite sorts, $F u n_{f_{1}, \ldots, f_{s}}$ can be expressed without equality. For a term $f\left(t_{1}, \ldots, t_{k}\right)$ (an atomic formula $Q\left(t_{1}, \ldots, t_{k}\right)$ ) introduce notations $f\left[t_{i_{1}}, \ldots, t_{i_{m}} ; \bar{\theta}\right]\left(Q\left[t_{i_{1}}, \ldots, t_{i_{m}} ; \bar{\theta}\right]\right)$, where $t_{i_{1}}, \ldots t_{i_{m}}$ are all complex immediate subterms and $\bar{\theta}$ is a sequence of all simple immediate subterms (i.e variables or constants) of $f\left(t_{1}, \ldots, t_{k}\right)$ (of $Q\left(t_{1}, \ldots, t_{k}\right)$ ). Then for any complex term $f\left[t_{1}, \ldots t_{m} ; \bar{\theta}\right]$ define a formula $\mathrm{val}_{f\left[t_{1}, \ldots t_{m} ; \bar{\theta}\right]}(v)$ as follows. If all immediate subterms are simple then $v a l_{f[; \bar{\theta}]}(v) \equiv v a l_{f}(\bar{\theta}, v)$, where $v$ is a fresh variable. Otherwise define it as

$v a l_{f\left[t_{1}, \ldots, t_{m} ; \bar{\theta}\right]}(v) \equiv \exists v_{1} \ldots v_{m}\left(\operatorname{val}_{t_{1}}\left(v_{1}\right) \wedge \ldots \wedge v a l_{t_{m}}\left(v_{m}\right) \wedge v a l_{f}\left(v_{1}, \ldots, v_{m}, \bar{\theta}, v\right)\right)$, where $v$ is a fresh variable.

Translate $[[\mathcal{M}]]$ into the formula in the new vocabulary, where all functional symbols $f$ are replaced by predicates $v a l_{f}$, as follows. Replace any atomic formula $Q\left[t_{1}, \ldots, t_{m} ; \bar{\theta}\right]$ with $\exists v_{1}, \ldots v_{m}\left(\operatorname{val}_{t_{1}}\left(v_{1}\right) \wedge \ldots \wedge v a l_{t_{m}}\left(v_{m}\right) \wedge Q\left(v_{1}, \ldots, v_{m}, \bar{\theta}\right)\right.$. Replace any atomic formula $t=s$, where $t, s$ are complex terms, with $\exists v\left(v a l_{t}(v) \wedge v a l_{s}(v)\right)$. Replace any atomic formula $t=r$, where $t$ is complex and $r$ is simple term, with $\left.v a l_{t}(v)\right|_{v \mapsto r}$ (i.e $r$ is substituted into $v$ in $\left.\operatorname{val}_{s}(v)\right)$.

Given that $\left[[T R]_{\text {frame }}\right.$ and $\left[[T R]_{\text {rules }}\right.$ are now in the relational vocabulary, further translation is performed as follows: replace all quantifiers over finite sorts with conjunctions and disjunctions over elements of the sort. Then, the first and second conditions of the definition of monodic rules ensure that, in the resulting formula, we have no more than one free variable in subformulae under the $\bigcirc$ operator in $\left[[T R]_{\text {rules }}\right.$ and in the Persists subformulae in $\llbracket T R \rrbracket_{\text {frame }}$. It follows that the resulting formulae $\left[T R \rrbracket_{\text {rules }}^{*}\right.$ and $\left[[T R]_{\text {frame }}^{*}\right.$ are monodic.

Consider subformulae of the form $v_{i}=t_{i}(\bar{x})$ in the $\Phi_{f}$ subformulae of $\left[[T R]_{\text {frame }}\right.$ and $\llbracket T R \rrbracket_{\text {rules }}$. During the above translation into relational vocabulary all equalities in such subformulae were eliminated, except the case, when $t_{i}(\bar{x})$ is a constant, or a variable. If it is a constant, then according to the condition 3 of the definition of monodic rules, it can be only of a finite sort and the quantification of $v_{i}$ over this sort can be eliminated together with equality. If it is a variable, then subformula $v_{i}=x$ of $[T R]_{\text {rules }}$ is replaced with true, the quantifier over $v_{i}$ is removed and all occurences of $v_{i}$ are replaced by $x$. If $v_{i}=x$ is a subformula of $\Phi_{f}$ of $\left[[T R]_{\text {frame }}\right.$ then it is replaced with true and is used to remove all occurences of $x$ in $\Phi_{f}$. To illustrate last point consider as an example a formula, describing a frame condition:

$$
\square \forall v, w, z(\neg \exists x(v=3 \wedge w=x \wedge b(x))) \Rightarrow \operatorname{Persists}\left(\operatorname{val}_{f}(v, w, z)\right)
$$

One can eliminate $w=x$ getting an equivalent formula:

$$
\square \forall v, w, z((v \neq 3 \vee \neg b(w))) \Rightarrow \operatorname{Persist}\left(\operatorname{val}_{f}(v, w, z)\right)
$$

Thus after elimination of all such subformulae, we have final translation $\llbracket[\mathcal{M}]^{*}$ of the initial $[\mathcal{M} \rrbracket]$ which is monodic and contains no equality. Finally, straightforward 
but long argument shows that all stages of above translation preserve the "meaning" of temporal formulae and $[[\mathcal{M}]]^{*}$ and $[[\mathcal{M}]]$ have the same set of temporal models modulo renaming of functional symbols into relational ones.

\section{Where Are the Monodic ASMs?}

The notion of monodic ASM presented in the previous section is fairly restricted. The area of its applicability still needs to be understood better. In this section we present two examples of realistic distributed algorithms (protocols) which can be specified and verified via approach we have presented.

\subsection{FloodSet Algorithm}

We consider here a variant of the FloodSet algorithm with alternative decision rule (in terms of [29], p.105) designed for solution of the Consensus problem in the presence of crash (or fail-stop) failures.

The setting is as follows. There are $n$ processes, each having an input bit and an output bit. The processes work synchronously, run the same algorithm and use broadcast for communication. Some processes may fail and, from that point onward, such processes do not send any further messages. Note, however, that the messages sent by a process in the moment of failure may be delivered to an arbitrary subset of the processes. Crucially, there is a bound, $f$, on the number of processes that may fail.

The goal of the algorithm is to eventually reach an agreement, i.e. to produce an output bit, which would be the same for all non-faulty processes. It is required also that if all processes have the same input bit, that bit should be produced as an output bit.

This protocol (adapted from [29] ${ }^{1}$ ) is as follows.

- At the first round of computations, every process broadcasts its input bit.

- At every later round, a process broadcasts any value the first time it sees it.

- At every round the (tentative) output bit is set to the minimum value ever seen so far.

The correctness criteria for this protocol is that, eventually (actually, no later than in $f+2$ rounds) the output bits of all non-faulty processes will be the same.

Claim. The above FloodSet algorithm can be specified (naturally) as a monodic ASM $\mathcal{M}$. Its temporal translation $[[\mathcal{M}]]$ and correctness condition are in the decidable twovariable monodic (without equality) fragment of FOTL under finite domain semantics.

The detailed justification of this claim can be found in [14]. Here we present only one rule and its corresponding frame condition as examples. The vocabulary of ASMs here consists of (among others) unary predicate symbols Normal (dymanic), Failure (external) and Faulty (dynamic). Intuitively Failure $(x)=$ true at some moment means "processor denoted by $x$ fails" at that moment. Similarly for Faulty $(x) \cdot \operatorname{Normal}(x)$ means $x$

\footnotetext{
${ }^{1}$ The difference being that, in [29], every process knows the bound $f$ in advance and stops the execution of the protocol after $f+2$ rounds, producing the appropriate output bit. For generality, we consider the version where the processes do not know $f$ in advance and produce a tentative output bit at every round.
} 
is not faulty nor in a failure state. One of the transition rules is if $\operatorname{Normal}(x) \wedge$ Failure $(x)$ then $\operatorname{Faulty}(x) \wedge \neg \operatorname{Normal}(x)$.

Our translation gives, for frame conditions on Faulty (after simplification):

1.

$$
\begin{aligned}
\text { 1. } & \square \forall x, z \neg(\operatorname{Normal}(x) \\
\text { 2. } & \wedge \text { Failure }(x)) \rightarrow \text { Persists }(\text { Faulty }(x)) \\
\square \forall x, z \neg(\operatorname{Normal}(x) & \wedge \text { Failure }(x)) \rightarrow \operatorname{Persist}(\neg \text { Faulty }(x))
\end{aligned}
$$

and

\subsection{Cache Coherence Protocols}

Another class of protocols which can be specified via monodic ASMs, and for which correctness can be reduced to the validity of monodic temporal formulae, is the class of cache coherence protocols [21]. These are designed to provide data consistency betwen caches of different processors in shared-memory multiprocessor systems, i.e. to ensure that any copies of the same memory block in the caches of different processors are identical. Abstracting from the low-level implementation details of read, write and syncronisation primitives, cache coherence protocols $[21,10]$ can be seen as describing the families of identical finite state machines (each being cache controller of a processor) together with a simple form of communication: when one machine makes a transition (an action) $a$, the the other machines are required to do complementary transition (a re-action) $\bar{a}$. Correctness can then be expressed in terms of co-occurences some states in which, for example, the processors are allowed to change the content of their caches.

In [13] we have shown that theorem proving in monodic temporal fragments can be used for the verification of cache coherence protocols for an arbitrary number of processors. Here, we illustrate the point that natural ASM specifications of finite state based models of cache coherence protocols can be translated into temporal monodic specifications by the above translation procedure. As an example we consider "Synapse N+1" protocol which is particularly simple, consisting of only 3 states, 2 actions (transitions), Read and Write and two re-actions (complementary transitions) $\overline{R e a d}$ and $\overline{\text { Write }}$. The full ASM specification of this protocol (for an arbitrary number of processors) together with comments on temporal translation and verification can be found in [14]. For the moment, we simply note that, in the ASM specification of that protocol, we assume

- the elements of the domain represent cache controllers of different processors;

- states of cache controllers are represented by unary predicates holding or not on the corresponding elements of the domain, e.g. $P(x)$ means "automaton $\mathrm{x}$ is in state $P$ ";

- use of only two (external) predicates Read and Write for actions, while reactions $\overline{\text { Read }}$ and $\overline{\text { Write }}$ are expressed in terms of these two.

The ASM specification is monodic, except for one integrity constraint that uses an equality, expressing the fact that no more than one processor can act at every moment of time. The correctness conditions have a form

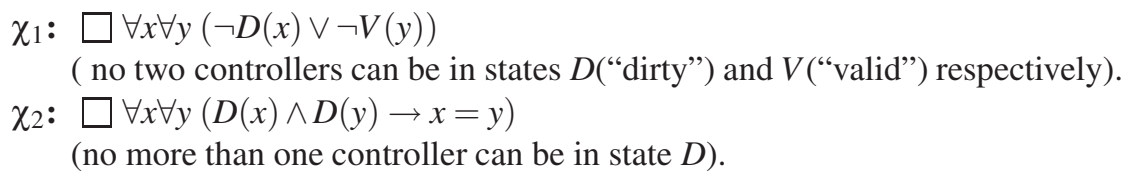


In general, since the obtained temporal formulae are monodic, we must consider the effect of the use of the equality predicate. It is known that even severely restricted classes of monodic formulae with equality may have a highly undecidable validity checking problem [8]. However, as is shown in [13] the proof methods for the fragment without equality [4] may be used for the proof of the above correctness condition $\chi_{1}$ (and such like). As to the $\chi_{2}$, the equality may be abstracted away, by renaming the entire formula by a propositional letter and adding a corresponding $\square$-closed equivalence to the specification. After that the same procedure proves the validity.

\section{Related Work}

We have learned from one of the referees that in [33] Antje Nowack has suggested to use monodic fragments of FOTL to verify ASMs. In particular, he proposed the definition of guarded ASM and has shown that verification of the properties of such ASMs expressed in the guarded monodic fragment of FOTL is decidable. A reduction to the finite satisfiability problem of guarded monodic fragment is used.

It turns out that our work (done independently) is very much in spirit of [33]. We formulated restrictions on ASM which ensure that temporal translation falls into monodic fragment, which is, in general, undecidable, but finitely axiomatizable and hence semidecidable. Technically, our definition of monodic ASM is less restrictive than that of guarded ASMs. On the other hand we can only guarantee existence of semi-decision procedure for monodic ASM (and suitable correctness conditions) as opposed to decision procedure for guarded ASMs (and suitable correctness conditions). However, checking whether the result of our temporal translation is in a known decidable fragment of FOTL is straightforward, so one can apply a decision procedure if it is the case. If the translation is outside known decidable fragment one can apply incomplete methods fo verification. We have illustrated both these cases with the examples.

\section{Conclusion}

We have shown how to faithfully translate ASM specifications into the first-order temporal logic (FOTL) and have defined restrictions on ASM specifications (programs) which ensure that temporal translation is in a 'good' (monodic) fragment of FOTL. This allows us to use temporal translations for (semi-)automatic verifications of restricted ASM specifications either by decision procedures [7], or by theorem proving for restricted fragments of FOTL [4].

Design of effective automatic algorithms, where possible, is an important future development, as is an in-depth analysis of the applicability of monodic ASMs.

\section{References}

1. Abstract State Machines: A Formal Method for Specification and Verification. [http: //www. eecs.umich.edu/gasm/].

2. A. Blass and Y. Gurevich. Abstract State Machines Capture Parallel Algorithms. ACM Transactions on Computational Logic, 2003. 4(4), October 2003. 
3. E. Börger. High Level System Design and Analysis using Abstract State Machines. In Current Trends in Applied Formal Methods (FM-Trends 98), number 1641 in LNCS, pages 1-43. Springer-Verlag, 1999.

4. J. Brotherston, A. Degtyarev, M. Fisher, and A. Lisitsa. Implementing Invariant Search via Temporal Resolution. In Proc. LPAR-2002. Springer Verlag, 2002.

5. E. Clarke, O. Grumberg, and D. Peled. Model Checking. MIT Press, Dec. 1999.

6. A. Degtyarev and M. Fisher. Towards First-Order Temporal Resolution. In Proc. KI-2001, pages 18-32. Springer Verlag, 2001. Volume 2174 of LNAI.

7. A. Degtyarev, M. Fisher, and B. Konev. Monodic temporal resolution. Proc. CADE'03, Springer.

8. A. Degtyarev, M. Fisher, and A. Lisitsa. Equality and Monodic First-Order Temporal Logic. Studia Logica, (72) 2002, PP 147-156.

9. G. Del Castillo and K. Winter. Model Checking Support for the ASM High-Level Language. In Proc. TACAS, volume 1785 of LNCS, pages 331-346. Springer-Verlag, 2000.

10. G. Delzanno, Constraint-based verification in Parametrized Cache Coherence Protocols, to appear in Formal Methods in System Design

11. E. A. Emerson. Temporal and Modal Logic. In Handbook of Theoretical Computer Science, pages 996-1072. Elsevier, 1990.

12. M. Fisher, C. Dixon, and M. Peim. Clausal Temporal Resolution. ACM Transactions on Computational Logic, 2(1):12-56, Jan. 2001.

13. M. Fisher, A. Lisitsa. Deductive Verification of Cache Coherence Protocols. In Proceedings of the 3rd Workshop on Automated Verification of Critical Systems AVoCS'03, Southampton, UK, April 2003, pp 177-186

14. M. Fisher, A. Lisitsa. Temporal Verification of Monodic Abstract State Machines Technical Report ULCS-03-011, University of Liverpool Department of Computer Science, April 2003, avaialable at http: / / www. csc. Iiv.ac.uk/research/techreports

15. A. Gargantini and E. Riccobene. Encoding Abstract State Machines in PVS. In Abstract State Machines: Theory and Applications, volume 1912 of LNCS. Springer-Verlag, 2000.

16. Y. Gurevich. Evolving Algebras 1993: Lipari Guide. In Specification and Validation Methods, pages 9-36. Oxford University Press, 1995.

17. Y. Gurevich. Sequential Abstract State Machines Capture Sequential Algorithms. ACM Transactions on Computational Logic, 1(1):77-111, July 2000.

18. Y. Gurevich. Logician in the land of OS: Abstract State Machines at Microsoft. In Proc. 16th Annual IEEE Symposium on Logic in Computer Science (LICS), pages 129-136. IEEE Computer Society, 2001.

19. U. Glässer and Y. Gurevich and M. Veanes. Universal Plug and Play Models. Technical Report MSR-TR-2001-59, Microsoft Research, June 2001.

20. Y. Gurevich and W. Schulte and M. Veanes. Toward Industrial Strength Abstract State Machines. Technical Report MSR-TR-2001-98, Microsoft Research, October 2001.

21. J. Handy. The Cache memory Book. Academic Press, 1993.

22. I. Hodkinson Monodic Packed Fragment with Equality is Decidable. Studia Logica, (72) 2002, pp 185-197.

23. I. Hodkinson, F. Wolter, and M. Zakharyashev. Decidable Fragments of First-Order Temporal Logics. Annals of Pure and Applied Logic, 2000.

24. U. Hustadt and B. Konev. TRP 2.0: A Temporal Resolution Prover. Proc. CADE'03, Springer.

25. U. Hustadt and R. A. Schmidt. Formulae which Highlight Differences between Temporal Logic and Dynamic Logic Provers. In Issues in the Design and Experimental Evaluation of Systems for Modal and Temporal Logics, Technical Report DII 14/01, pages 68-76. Dipartimento di Ingegneria dell'Informazione, Unversitá degli Studi di Siena, 2001. 
26. U. Hustadt and R. A. Schmidt. Scientific Benchmarking with Temporal Logic Decision Procedures. In Proc. KR'2002, pages 533-544. Morgan Kaufmann, 2002.

27. M. Huth and M. Ryan. Logic in Computer Science. Cambridge University Press, 2000.

28. B. Konev, A. Degtyarev, C. Dixon, M. Fisher, and U. Hustadt. Towards the Implementation of First-Order Temporal Resolution: the Expanding Domain Case. In Proceedings of TIMEICTL 2003, July 2003, Cairns, Queensland, Australia. IEEE CS Press.

29. N. Lynch. Distributed Algorithms. Morgan Kaufmann Publishers, San Mateo, CA, 1996.

30. Z. Manna and the STeP group. Step: Deductive-algorithmic verification of reactive and realtime systems. In International Conference on Computer Aided Verification (CAV), volume 1102 of LNCS. Springer-Verlag, 1996.

31. Z. Manna and A. Pnueli. The Temporal Logic of Reactive and Concurrent Systems: Specification. Springer-Verlag, New York, 1992.

32. Z. Manna and A. Pnueli. Temporal Verification of Reactive Systems: Safety. Springer-Verlag, New York, 1995.

33. A. Nowack. Deciding the Verification Problem for Abstract State Machines. In Proceedings of ASM 2003, LNCS, 2589: 341-371, 2003.

34. R. Reiter. The frame problem in the situation calculus: A simple solution (sometimes) and a completeness result for goal regression. In AI and Mathematical Theory of Computation: Papers in Honor of John McCarthy, pages 359-380. Academic Press, 1991.

35. J. Richardson, A. Smaill, and I. Green. System description: Proof planning in higher-order logic with $\lambda$ clam. LNCS, 1421:129-131, 1998.

36. G. Schellhorn and W. Ahrendt. Reasoning about Abstract State Machines: The WAM Case Study. Journal of Universal Computer Science, vol. 3, no. 4 (1997), 377-413.

37. A. P. Sistla and E. M. Clarke. Complexity of Propositional Linear Temporal Logics. ACM Journal, 32(3):733-749, July 1985.

38. M. Spielmann. Model Checking Abstract State Machines and Beyond. In Abstract State Machines: Theory and Applications, volume 1912 of LNCS, pages 323-340. Springer-Verlag, 2000.

39. K. Winter. Towards a Methodology for Model Checking ASM: Lessons Learned from the FLASH Case Study. In Abstract State Machines: Theory and Applications, volume 1912 of LNCS, pages 341-360. Springer-Verlag, 2000.

40. M. Wooldridge. Reasoning about Rational Agents. MIT Press, 2000.

41. F. Wolter and M. Zakharyaschev. Axiomatizing the Monodic Fragment of First-Order Temporal Logic. Annals of Pure and Applied logic, 118:133-145, 2002. 